\title{
Study of the functionality of the Helicobacter pylori trans-translation components SmpB and SsrA in an heterologous system
}

\author{
Marie Thibonnier1,2, Sylvie Aubert ${ }^{1}$, Chantal Ecobichon 1,3 and Hilde De Reuse*1
}

\begin{abstract}
Background: Trans-translation is a ubiquitous bacterial quality control-mechanism for both transcription and translation. With its two major partners, SsrA a small stable RNA and the SmpB protein, it promotes the release of ribosomes stalled on defective mRNAs and directs the corresponding truncated proteins to degradation pathways. We have recently shown that trans-translation is an essential function in the gastric pathogen Helicobacter pylori. Our results suggested that some properties of the H. pylori trans-translation machinery distinguishes it from the well known system in E. coli. Therefore, we decided to test the functionality of the SmpB and SsrA molecules of H. pylori in the E. coli heterologous system using two established phenotypic tests.

Results: H. pylori SmpB protein was found to successfully restore the E. coli $\triangle s m p B$ mutant growth defect and its capacity to propagate $\lambda i m m^{P 22}$ phage. We showed that in E. coli, H. pylori SsrA (Hp-SsrA) was stably expressed and maturated and that this molecule could restore wild type growth to the E. coli $\triangle s s r A$ mutant. Hp-SsrA mutants affected in the ribosome rescue function were not able to restore normal growth to $E$. coli $\triangle s s r A$ supporting a major role of ribosome rescue in this phenotype. Surprisingly, $\mathrm{Hp}$-SsrA did not restore the phage $\lambda i m m^{\text {P22 }}$ propagation capacity to the E. coli $\triangle s s r A$ mutant.

Conclusions: These data suggest an additional role of the tag sequence that presents specific features in Hp-SsrA. Our interpretation is that a secondary role of protein tagging in phage propagation is revealed by heterologous complementation because ribosome rescue is less efficient. In conclusion, tmRNAs present in all eubacteria, have coevolved with the translational machinery of their host and possess specific determinants that can be revealed by heterologous complementation studies.
\end{abstract}

\section{Background}

Trans-translation is a quality-control mechanism that is ubiquitous in bacteria and involves two activities [1-3]. First, trans-translation favors the rescue of ribosomes stalled on defective or damaged mRNAs (lacking a stop codon) through the restart of translation. Second, transtranslation functions to direct incomplete peptides to degradation by the addition of a specific tag [4]. Transtranslation is generally non-essential and requires two factors: SsrA, a small stable structured RNA (also called tmRNA) that acts both as a tRNA by its alanylated tRNA-

\footnotetext{
* Correspondence: hdereuse@pasteur.fr

1 Institut Pasteur, Unité P. Pathogenèse de Helicobacter, 28 rue du Dr. Roux, 75724 Paris Cedex 15 France

Full list of author information is available at the end of the article
}

like domain (TLD) and as a mRNA-like domain (MLD) [4] and its protein cofactor, SmpB.

The length and sequence of the trans-translation appended peptide tag varies with the bacterial species (between 8 and 35 amino acids) [5]. Mostly studied in $E$. coli, the tag encoded by SsrA is sufficiently informative to target any trans-translated proteins to degradation pathways [4]. The phenotypes of mutants deficient in this process depend on the species examined and are related to environmental adaptation, differentiation, stress response or virulence (for a review see [6]). Growing evidence indicates that trans-translation tagging targets specific substrates and therefore plays a regulatory role in organisms such as Caulobacter crescentus [7,8] Yersinia pseudotuberculosis [9], Helicobacter pylori [10] or Streptomyces coelicolor [11]. 
In E. coli, numerous phenotypes were associated with the deficiency of trans-translation, among which a slight enhancement of the doubling time that was observed even under normal growth conditions [12]. One of the tools used to characterize the SsrA determinants in vivo was the dependence on trans-translation of the growth of the hybrid bacteriophage $\lambda i m m^{\mathrm{P} 22}$ in E. coli [13-15]. This phage is a hybrid between the $E$. coli lambda phage and the Salmonella P22 phage and is specific for E. coli. E. coli strains defective in trans-translation display a characteristic phenotype termed "Sip" (for selectively inhibits of $\lambda i m m$ P22) [13]. Indeed, the frequency of infection by $\lambda i m m^{\mathrm{P} 22}$ is 10,000 -fold lower in $\Delta s m p B$ or $\Delta s s r A$ E. coli mutants as compared to that in the corresponding parental strain $[13,16]$. The precise molecular basis of the phage plating defect in trans-translation-deficient cells is not yet understood. The impact of SsrA point mutations on $\lambda \mathrm{imm}^{\mathrm{P} 22}$ growth in E. coli was first analyzed by Withey and Friedman [14] who showed (i) that charging of tmRNA with Ala was essential and, (ii) that degradation of proteins tagged by tmRNA was only required to achieve optimal levels of phage growth. A more recent study challenged these conclusions and demonstrated that $\lambda i m m^{\mathrm{P} 22}$ propagation in $E$. coli is exclusively dependent on ribosome recycling functions of trans-translation and not on its proteolysis targeting activity [15].

We have recently investigated the role of trans-translation in Helicobacter pylori [10]. H. pylori is a bacterial pathogen that colonizes the stomach of half of the human population and is strongly adapted to persist and multiply under stressful conditions such as low $\mathrm{pH}$. Colonization of the stomach by $H$. pylori is associated with several gastric pathologies ranging from gastritis, peptic ulcer to adenocarcinoma [17]. We demonstrated that ribosome rescue by trans-translation is essential for in vitro growth of $H$. pylori. Interestingly, stress resistance and natural competence were strongly affected in $H$. pylori strains carrying a mutated $t m$ RNA tag sequence [10]. While the overall structure of $H$. pylori SsrA is conserved, the tag sequence significantly differed from that of $E$. coli and our mutagenesis study revealed both identical and different properties as compared to its E. coli homolog [10]. To investigate further these differences using a model organism, we decided to study the H. pylori SmpB and SsrA expressed in the $E$. coli heterologous system.

\section{Results}

Functional complementation of an E. coli smpB deletion mutant by Hp-SmpB

To examine the functionality of the SmpB protein of $H$. pylori $(\mathrm{Hp}-\mathrm{SmpB})$ in $E$. coli, the corresponding gene hp1444 was amplified from H. pylori strain 26695 and cloned into pILL2150 under control of an inducible promoter, to generate pILL786 (Table 1). This plasmid was transformed into E. coli wild type strain MG1655 and its isogenic $\triangle \operatorname{smp} B$ mutant [18] (Table 1 and 2). Expression of Hp-SmpB in E. coli was verified by western blot in the $\triangle s m p B$ mutant using antibodies raised against purified E.coli $\mathrm{SmpB}$. Hp-SmpB was detected, its synthesis was strongly enhanced upon addition of IPTG and was overexpressed in comparison with the $E$. coli endogenous SmpB protein, Ec-SmpB (Figure 1).

The efficacy of propagation of the hybrid phage $\lambda \mathrm{imm}^{\mathrm{P} 22}$ [13] was measured on different strains. Table 3 presents the relative efficiency of plating (EOP) of each strain in comparison with that of the wild type parental strain. Phage propagation on strain MG1655 $\Delta s m p B$ containing the empty vector pILL2150 was, as expected, strongly affected with an EOP of $1.3 \times 10^{-5}$ (Table 3). Relative EOP of strain MG1655 $\Delta s m p B$ pILL786 in the presence of IPTG, expressing Hp-SmpB is close to 1 (Table 3). This result demonstrated that $\mathrm{Hp}-\mathrm{SmpB}$ is active in E. coli and efficiently complemented the phage propagation defect phenotype. In addition, the growth defect of MG1655 $\triangle \operatorname{smp} B$ mutant was analyzed with or without Hp-SmpB. Under our test conditions, MG1655 $\Delta s m p B$ mutant presented a doubling time that was about twice that of the wild type strain and was restored to wild type growth in the presence of $\mathrm{Hp}-\mathrm{SmpB}$ expressed by pILL786 (Figure 2 and Table 3). This indicated that Hp$\mathrm{SmpB}$ is able to replace Ec-SmpB functions during transtranslation in E. coli.

\section{Expression and maturation of $\mathrm{Hp}$-SsrA in E. coli}

To evaluate the heterologous complementation capacity of Hp-SsrA in E. coli, we constructed pILL788 and pILL2318 carrying the $s s r A$ gene of $H$. pylori under control of a promoter on high copy and low copy number plasmids, respectively (Table 1). Plasmids pILL788 and pILL2318 expressing wild type Hp-SsrA were trans-

\begin{tabular}{|c|c|c|c|c|}
\hline Lane & 1 & 2 & 3 & 4 \\
\hline $\begin{array}{l}\text { E. coli } \\
\text { strain }\end{array}$ & MG1655 & $\begin{array}{c}\text { MG1655 } \\
\Delta s m p B\end{array}$ & \multicolumn{2}{|c|}{$\begin{array}{c}\text { MG1655 } \\
\Delta s m p B \text { plLL786 }\end{array}$} \\
\hline $\mathrm{SmpB}^{E c}$ & + & - & - & - \\
\hline $\mathrm{SmpB}^{H p}$ & - & - & + & + \\
\hline + IPTG (1 mM) & - & - & - & + \\
\hline MW $20 \mathrm{KDa}-$ & & & & \\
\hline
\end{tabular}

Figure 1 Detection of SmpB in E. coli. Detection of SmpB protein in E. coli was performed by western blot with an E. coli SmpB polyclonal antibody. Lane 1: wild type E. colistrain (predicted MW SmpB ${ }^{E c}=18,125$ Da), lane 2: $\triangle s m p B$ E. coli mutant. Lanes 3-4: SmpBHp detection in a $\triangle S m p B E$. coli mutant carrying the inducible vector pILL786 expressing the $s m p B^{H p}$ gene (predicted MW SmpB ${ }^{H p}=17,682 \mathrm{Da}$ ), with or without induction with 1 mM IPTG, respectively. Calibrated amounts of crude bacterial extracts were separated by SDS-15\% PAGE. MW: molecular weight. 
Table 1: Plasmids used in this study

\begin{tabular}{|c|c|c|}
\hline Plasmids & Relevant features & Reference \\
\hline pEXT21 & low copy number E. coli vector & {$[25]$} \\
\hline pILL2318 & H. pylori ssrAWT cloned into pEXT21 & This study \\
\hline pILL2150 & $\begin{array}{l}\text { high copy number H. pylori/E. coli shuttle } \\
\text { vector }\end{array}$ & [24] \\
\hline plLL2334 & E. coli ssrAWT cloned into pILL2150 & This study \\
\hline pILL786 & $\begin{array}{l}\text { hp1444 encoding } \mathrm{Hp}-\mathrm{SmpB} \text { cloned into } \\
\text { plLL2150 }\end{array}$ & This study \\
\hline pILL788 & H. pylori ssrAWT cloned into pILL2150 & [10] \\
\hline pILL791 & H. pylori ssrADD cloned into pILL2150 & {$[10]$} \\
\hline pILL792 & H. pylori ssrAresume cloned into pILL2150 & [10] \\
\hline pILL793 & H. pylori ssrAwobble cloned into pILL2150 & {$[10]$} \\
\hline plLL794 & H. pylori ssr $A^{S m p B}$ cloned into pILL2150 & {$[10]$} \\
\hline plLL2328 & H. pylori ssrA $A^{S T O P}$ cloned into plLL2150 & {$[10]$} \\
\hline
\end{tabular}

formed into both MG1655 wild type and $\Delta s s r A$ strains (Table 2). The expression of Hp-SsrA was examined by northern blot with total RNA extracted from different $E$. coli strains and from the H. pylori 26695 strain (Figure 3). A $300 \mathrm{nt}$ long riboprobe was chosen in the region of HpSsrA displaying homology with Ec-SsrA. A band of $386 \mathrm{nt}$ that matches the size of the mature Hp-SsrA was detected in the RNA samples extracted from $E$. coli MG1655 $\Delta s s r A$ pILL788 and MG1655 $\Delta s s r A$ pILL2318 strains (Figure 3). As expected, the amount of Hp-SsrA is weaker when expressed from the low copy plasmid pILL2318 than from pILL788. With RNA extracted from $H$. pylori strain 26695, we observed an intense band of the same size that was absent in samples extracted from MG1655 $\Delta s s r A$ containing pILL2150, the empty vector (Figure 3). A faint band corresponding to mature Ec-SsrA (363 nt) was detected in E. coli MG1655 wild type strain. This indicates that in E. coli, Hp-SsrA is expressed and correctly maturated.

\section{Analysis of the functionality of Hp-SsrA in E. coli}

The capacity of Hp-SsrA to complement the phage propagation defect of an E. coli strain deficient in SsrA was examined. The EOP of strain MG1655 $\Delta s s r A$ pILL2150 (empty vector) was $2.6 \times 10^{-5}$ as expected (Table 3 ). Surprisingly, the presence of pILL788 expressing processed Hp-SsrA in strain MG1655 $\Delta s s r A$ did not restore the capacity to propagate phage $\lambda i m m^{\mathrm{P} 22}$ (Table 3 ). This showed that Hp-Ssr A is not able to replace Ec-SsrA in this phenotypic test. It was controlled that phage $\lambda \mathrm{imm}^{\mathrm{P} 22}$ propagation was restored in strain MG1655 $\Delta s s r A$ pILL2334 expressing wild type Ec-SsrA on a plasmid.

Under our test conditions, the doubling time of $E$. coli $\triangle s s r A$ mutant was twice that of the wild type strain (Figure 2). Interestingly, wild type growth was restored in the E. coli $\triangle s s r A$ mutant complemented with plasmid pILL788 that expresses high levels of Hp-SsrA (Figure 2) but not with plasmid pILL2318 that expresses low levels of $\mathrm{Hp}$-SsrA. As a control, wild type growth was also observed with strain MG1655 $\Delta s s$ A pILL2334 expressing wild type Ec-SsrA. This indicated that Hp-SsrA is functional to rescue the growth defect of $E$ coli $\Delta s s r A$ but is not able to restore the phage propagation deficiency. We then wanted to understand further the functional basis of the partial functionality of Hp-SsrA in E. coli. 
Table 2: E. coli strain used in this study.

\begin{tabular}{|c|c|c|}
\hline Strains & ssr $A$ and smpB alleles & $\begin{array}{l}\text { Plasmids } \\
\text { [antibiotic resistance] }\end{array}$ \\
\hline MG1655 pILL2150 & $s m p B^{E C} s s r A^{E c / p l L L} 2150$ & multicopy $[\mathrm{Cm}]$ \\
\hline MG1655 pEXT21 & smpBEc ssrAEc/pEXT21> & low copy $[S p]$ \\
\hline MG1655 $\Delta s m p B$ plLL2150 & $\Delta s m p B^{E C} s s r A^{E c} / \mathrm{plLL} 2150$ & multicopy $[\mathrm{Cm}]$ \\
\hline MG1655 $\Delta s m p B$ plLL786 & $\Delta s m p B^{E C} s s r A^{E C /} /$ ILL2150 with $s m p B^{H p}$ & multicopy $[\mathrm{Cm}]$ \\
\hline MG1655 $\Delta$ ssrA plLL2150 & $s m p B^{E c} \Delta s s r A^{E c / p l L L 2150}$ & multicopy $[\mathrm{Cm}]$ \\
\hline MG1655 $\Delta$ ssrA plLL2334 & $\begin{array}{l}\text { smpBEc } \triangle s s r A E c / \text { pILL } 2334 \\
\text { ssrAEc-WT }\end{array}$ & multicopy $[\mathrm{Cm}]$ \\
\hline MG1655 $\Delta s s r A$ plLL788 & $\begin{array}{l}\quad s m p B E c \Delta s s r A E c / p I L L 2150 \\
\text { ssrAHp-WT }\end{array}$ & multicopy $[\mathrm{Cm}]$ \\
\hline MG1655 $\Delta s s r A$ plLL2318 & $\begin{array}{l}\quad s m p B E c \Delta s s r A E c / p E X T 21 \\
s s r A H p-W T\end{array}$ & low copy [Sp] \\
\hline MG1655 $\Delta s s r A$ plLL791 & $\begin{array}{l}\text { smpBEc } \Delta s s r A E c / p I L L 2150 \\
s s r A H p-D D\end{array}$ & multicopy $[\mathrm{Cm}]$ \\
\hline MG1655 $\Delta s s r A$ plLL792 & $\begin{array}{l}\text { smpBEc } \triangle s s r A E c / p I L L 2150 \\
\text { ssrAHp-resume }\end{array}$ & multicopy $[\mathrm{Cm}]$ \\
\hline MG1655 $\Delta s s r A$ plLL793 & smpBEc $\Delta s s r A^{E c} / p \mid L L 2150$ with ssrA $A^{H p-w o b b l e ~}$ & multicopy $[\mathrm{Cm}]$ \\
\hline MG1655 $\Delta$ ssrA plLL794 & $\begin{array}{l}\quad s m p B E c \Delta s s r A E c / p I L L 2150 \\
s s r A H p-s m p B\end{array}$ & multicopy $[\mathrm{Cm}]$ \\
\hline MG1655 $\Delta s s r A$ plLL2328 & $\begin{array}{l}\text { smpBEc } \Delta s s r A E c / p I L L 2150 \\
\text { ssrAHp-STOP }\end{array}$ & multicopy $[\mathrm{Cm}]$ \\
\hline
\end{tabular}

Analysis of the functionality of mutated $\mathrm{Hp}$-SsrA versions in E. coli

In a previous study, we constructed a series of five $H$. pylori SsrA mutants and evaluated in $H$. pylori their impact on trans-translation, survival and stress-response [10]. Characteristics of these mutations are summarized in Figure 4. Plasmids pILL793, pILL794 and pILL792 express mutant $\mathrm{Hp}$-SsrA that are unable to be alanylated on the TLD (SsrAwobble), to interact with $\mathrm{SmpB}$ (SsrA ${ }^{\mathrm{SmpB}}$ ) and to restart the translation on the MLD ( $\mathrm{SsrA}^{\text {resume}}$ ), respectively. Each of this mutation was found to be essential for growth of $H$. pylori [10]. When these plasmids were tested for complementation of the E. coli $\Delta s s r A$ mutant, neither phage propagation nor growth defective phenotypes was rescued (Figure 2 and Table 3 ).

In H. pylori, two mutations in the MLD of Hp-SsrA were found to be viable but affected the capacity of the corresponding mutant strains to resist to various stresses [10]. One mutation targets the terminal part of the tag sequence, the corresponding mutant gene $\mathrm{Hp}-\mathrm{SsrA}^{\mathrm{DD}}$ is 


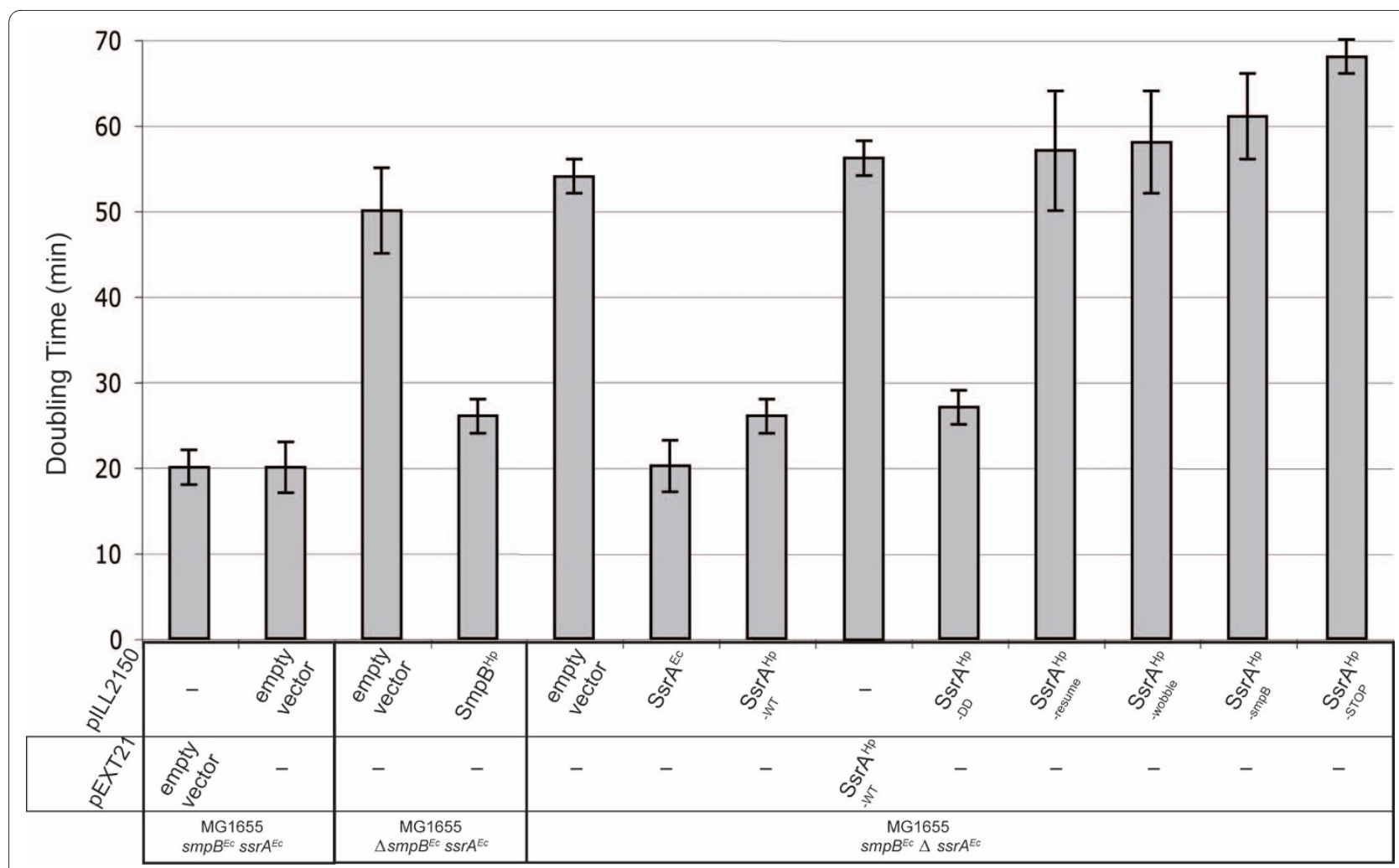

Figure 2 Doubling time of $E$. coli $\Delta s s r A$ or $\Delta s m p B$ mutants expressing SmpB ${ }^{H p}$ WT, SsrA ${ }^{H p}$ WT or mutants. Doubling times were calculated for E. coli strains expressing SmpBHp, SsrA Hp and different mutant versions of SsrAHp from plasmids. Doubling times ( $g$ values) correspond to the mean generation time. As a control, growth complementation of the E. coli $\triangle s s r A$ with $E c-s s r A$ is presented. Empty vector corresponds to a vector without insert.

carried by plasmid pILL791. This mutation was chosen because it was described to stabilize the trans-translated proteins in species like E. coli. In another mutant, HpSsrA ${ }^{\text {STOP }}$ (carried by pILL2328) two stop codons were introduced immediately downstream from the resume codon. As a consequence, Hp-SsrA ${ }^{\text {STOP }}$ adds a minimal tag (Ala-Val) to trans-translated proteins (Figure 4).

These two mutated Hp-SsrA versions did not restore the phage propagation capacity to the E. coli $\triangle s s r A$ mutant (Table 3). Interestingly, growth defect of the $E$. coli $\triangle s s r A$ mutant was restored to the wild type level by complementation with pILL791 expressing Hp-SsrA ${ }^{\text {DD }}$, and not with pILL2328 expressing Hp-SsrA STOP.

\section{Discussion}

Trans-translation is a bacterial ubiquitous mechanism of quality-control for protein and mRNA synthesis. We have recently shown that trans-translation is essential for in vitro growth of the gastric pathogen $H$. pylori [10] like in a few other human pathogens, Mycoplasma genitalium [19], Neisseria gonorrhoeae [20] or Haemophilus influenzae [21]. We also demonstrated that in H. pylori, the essential trans-translation function is ribosome rescue and that a single ribosomal translocation step is sufficient to promote release of stalled ribosomes [10]. Using differ- ent mutants of $H$. pylori ssr A, we found that under conditions of functional ribosome rescue, the tagging of transtranslated proteins was required for tolerance to both oxidative and antibiotic stresses and for effective natural competence. These data revealed for the first time that control of protein degradation through trans-translation is by itself central in the management of stress conditions and of competence and supports a regulatory role of trans-translation dependent protein tagging. Since we anticipate that this regulatory role of protein tagging is underestimated in E. coli and because we possessed a collection of well-defined Hp-SsrA mutant, we decided to explore the functionality of the $H$. pylori trans-translational components in E. coli.

Measurement of the $\lambda \mathrm{imm}^{\mathrm{P} 22}$ phage propagation is a classical test to evaluate the functionality of trans-translation in E. coli. As previously reported, both $\triangle s s r A$ and $\triangle s m p B$ E. coli mutants exhibit a 10,000-fold defect of phage propagation [14]. E. coli SsrA mutants present a slight growth defect, enhanced sensitivity to stress and to sub-inhibitory antibiotic concentrations. These phenotypes are complemented by $E$. coli SsrA variants that add a tag lacking some proteolytic determinants (f.i SsrADD). Therefore, these phenotypes are likely not to depend on proteolysis. 
Table 3: Ability of $H$. pylori SmpB and of wild type or mutant alleles of $s s r A^{H p}$ to support growth of $\lambda$ imm $^{\mathrm{P22}}$ in $E$. coli $\Delta s s r A$ or $\Delta s m p B$ deletion mutants and to restore the growth defect in $E$. coli $\Delta s s r A$ or $\Delta s m p B$ mutants

\begin{tabular}{|c|c|c|c|}
\hline Strains & ssrA or smpB alleles & EOP§ & $\begin{array}{l}\text { Growth defect restoration in E. coli } \Delta s m p B \text { or in } \\
\text { E. coli } \Delta \text { ssrA }\end{array}$ \\
\hline MG1655 & smpBEc ssrAEc & 1 & - \\
\hline $\begin{array}{l}\text { MG1655 } \Delta s m p B \\
\text { pILL2150 }\end{array}$ & $\triangle s m p B^{E c} s s r A^{E c}$ & $1.3 \times 10^{-5}$ & no \\
\hline $\begin{array}{l}\text { MG1655 } \triangle s m p B \\
\text { pILL786 }\end{array}$ & $\Delta s m p B^{E C} s s r A^{E C} / s m p B^{H p}$ & 0.6 & yes \\
\hline $\begin{array}{l}\text { MG1655 } \Delta s s r A \\
\text { pILL2150 }\end{array}$ & $s m p B E c \triangle s s r A E c$ & $2.6 \times 10^{-5}$ & no \\
\hline $\begin{array}{l}\text { MG1655 } \Delta s s r A \\
\text { plLL2334 }\end{array}$ & $\begin{array}{r}s m p B E c \quad \triangle s s r A E c l \\
s s r A E c-W T\end{array}$ & 1 & yes \\
\hline MG1655 $\Delta s s r A$ plLL788 & $\begin{array}{c}\text { smpBEc } \quad \triangle s s r A E c / \\
s s r A H p-W T\end{array}$ & $5.0 \times 10^{-5}$ & yes \\
\hline MG1655 $\Delta s s r A$ plLL791 & $\begin{array}{r}\text { smpBEc } \quad \triangle s s r A E c / \\
s s r A H p-D D\end{array}$ & $1.6 \times 10^{-5}$ & yes \\
\hline $\begin{array}{l}\text { MG1655 } \triangle s s r A \\
\text { plLL2328 }\end{array}$ & $\begin{array}{l}\text { smpBEc } \quad \triangle s s r A E c l \\
\text { ssrAHp-STOP }\end{array}$ & $6.1 \times 10^{-5}$ & no \\
\hline MG1655 $\Delta s s r A$ pILL792 & $\begin{array}{l}\text { smpBEc } \triangle s s r A E c / \\
\text { ssrAHp-resume }\end{array}$ & $3.9 \times 10^{-5}$ & no \\
\hline MG1655 $\Delta$ ssrA pILL793 & $\begin{array}{l}\text { smpBEc } \triangle s s r A E c / \\
\text { ssrAHp-wobble }\end{array}$ & $2.3 \times 10^{-5}$ & no \\
\hline MG1655 SssrA pILL794 & $\underset{s s r A H p-s m p B}{s m p B E c \quad \triangle s s r A E c /}$ & $3.6 \times 10^{-5}$ & No \\
\hline
\end{tabular}

${ }^{\S} \mathrm{EOP}$ is the ratio of the titer of phage on a lawn of bacteria mentioned in the table divided by the titer of phage on a wild type bacterial lawn.

In a first test, H. pylori SmpB protein was found to successfully complement the E. coli $\triangle s m p B$ mutant for both phage propagation and growth despite only $34.6 \%$ identity between Ec-SmpB and Hp-SmpB. This showed that Hp-SmpB is able to interact with both the E. coli SsrA RNA and ribosomes to perform efficient trans-translation in E. coli.

Results with Hp-ssrA in E. coli revealed a more complex picture. First, we showed that upon expression in $E$. coli, Hp-SsrA is highly expressed and exhibits a size compatible with correct maturation. Indeed, Hp-SsrA and $\mathrm{Hp}-\mathrm{Ssr} \mathrm{A}^{\mathrm{DD}}$ restored a wild-type growth phenotype to an
E. coli $\Delta s s r A$ mutant indicating its functionality in E. coli. This result is in agreement with a minor role of the protein tagging step in the growth defect of Ecoli $\Delta s s r A$. Accordingly, we observed that the mutant versions of $\mathrm{Hp}$ SsrA that were affected in ribosome rescue ( $\mathrm{SsrA}^{\text {Resume, }}$ SsrA wobble and $\mathrm{SsrA}^{\mathrm{SmpB}}$ ) failed to complement the slow growth phenotype of E. coli $\triangle s s r A$. Unexpectedly, the HpSsrA ${ }^{\text {STOP }}$ mutant that contains an intact resume codon followed by two stop codons is not able to complement the E. coli $\triangle s s r A$ growth defect. This is surprising since in H. pylori, the SsrA ${ }^{\mathrm{STOP}}$ mutation is not essential for in vitro growth strongly suggesting that it is still effective in 


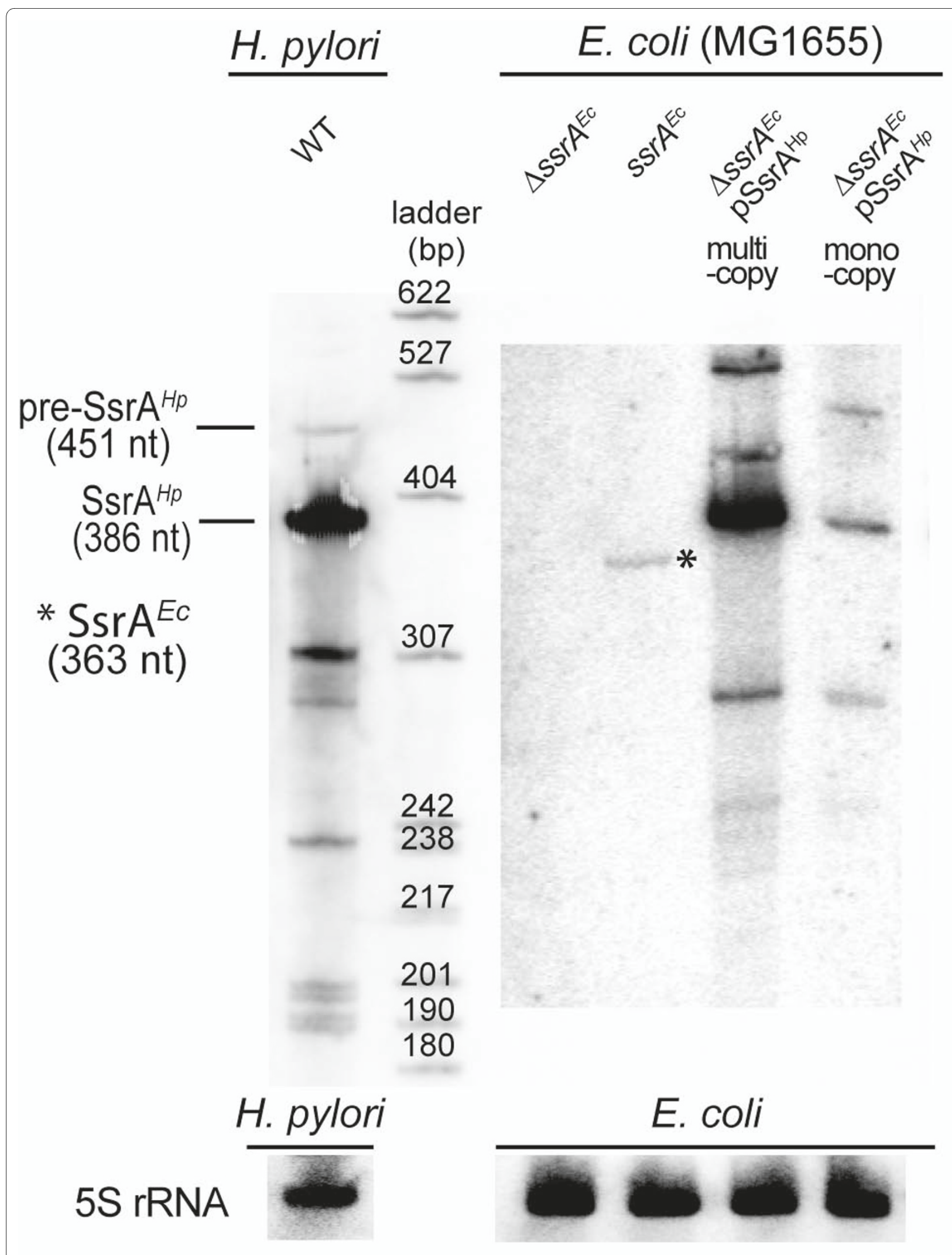

Figure 3 Detection of SsrA ${ }^{H p}$ expressed in H. pylori and from plasmids in E. coli. A SsrAHp riboprobe was used to perform northern blots and detect the SsrA Hp molecule in H. pylori and in E. coli wild type or $\triangle s s r A$ mutant strains. Pre-SsrAHpindicates a band with the size of non-maturated precursor of SsrA Hp. A faint band marked by a star corresponds to cross-hybridization with the SsrAEcthat is, as expected, absent in the E. coli $\triangle s s r A$ mutant. 


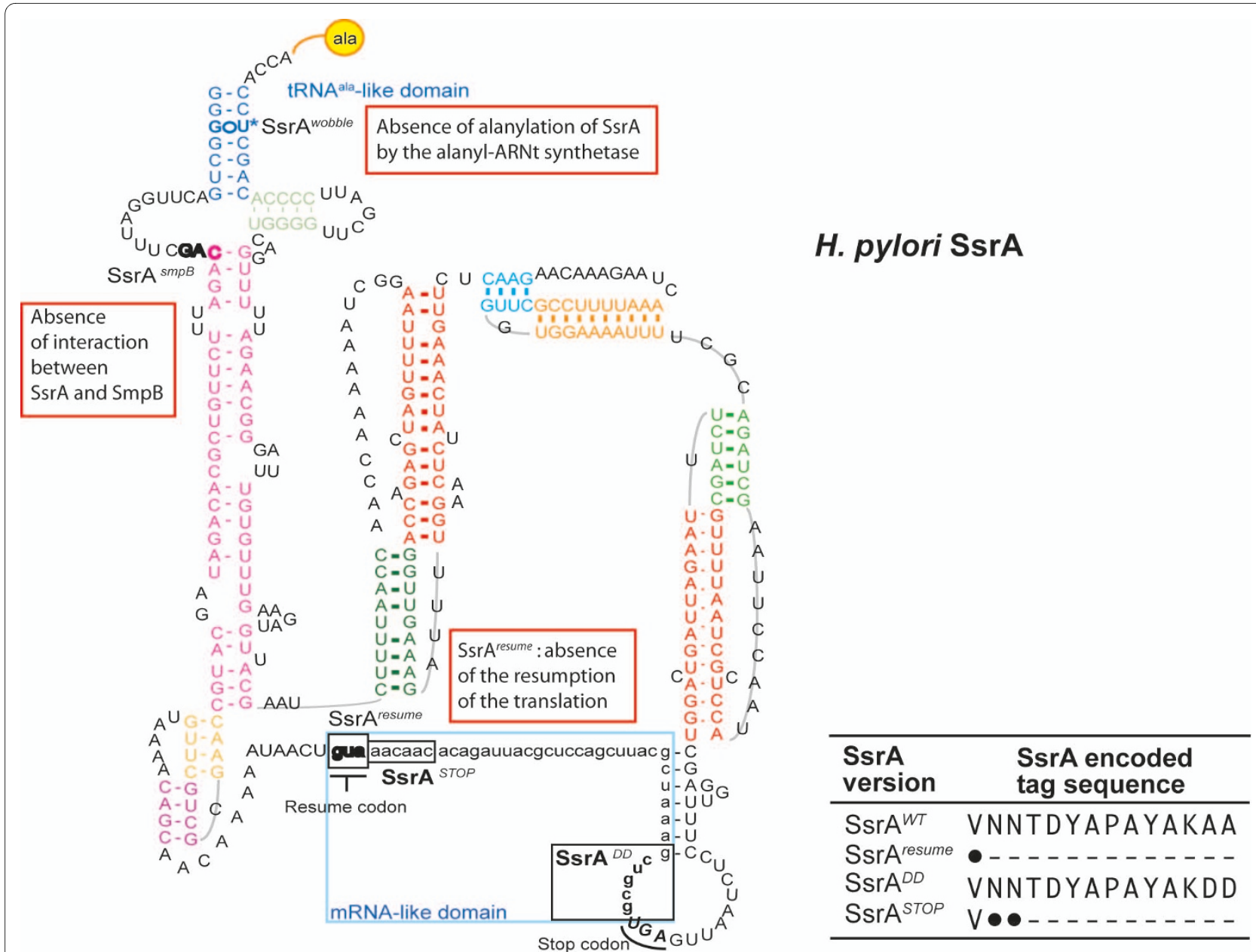

Figure 4 Mutations introduced into the H. pylori SsrA molecule. The model of the H. pylori mature SsrA molecule is after the tmRNA website http:/ /www.indiana.edu/ tmrna/. As described in [10], the SsrAwobble, SsrASmpB, SsrAresume mutations that abolish the trans-translation process are boxed in red. Mutations of the mRNA-like domain that affect the tag are also indicated. The amino acid sequence of the tag (wild type or mutant) appended to trans-translated proteins are listed in the table.

release of stalled ribosomes [10]. In a previous study [15], an equivalent mutation was introduced into E. coli SsrA, however only phage propagation phenotype is reported and no mention was made of the growth rate of this mutant. The most straightforward interpretation of our data is that trans-translation by $\mathrm{Hp}-\mathrm{SsrA}^{\mathrm{STOP}}$ in $E$. coli is not efficiently using the resume codon. Indeed, there are striking differences between Hp-SsrA and Ec-SsrA. In particular, the resume codon of Hp-SsrA is GUA encoding Valine and in E. coli, the resume codon GCA encodes Alanine (Figure 4) [5]. Replacement of the Ec-SsrA resume codon by GUA or GUC encoding Valine is functional in E. coli [22]. However, mass spectrometry analysis revealed that breakage of the peptide tag occurred frequently after certain residues like a Valine encoded by GUA and that these SsrA-tag added to proteins are ineffective in growth competition with $\Delta s s r A$ mutants [22]. Therefore, we hypothesize that the GUA resume codon of
$\mathrm{Hp}-\mathrm{SsrA}$ is a poor resume codon for trans-translation in $E$. coli and that additional downstream sequence compensate for this deficiency. As a consequence, the introduction of two stops immediately after the resume codon as in the Hp-SsrA ${ }^{\mathrm{STOP}}$ mutant might render this compensation impossible and translation restart ineffective. These data emphasize the strict constraints on SsrA sequence to achieve ribosome rescue in a given organism.

The functionality of Hp-SsrA in E. coli was also examined using the phage $\lambda \operatorname{imm}^{\mathrm{P} 22}$ propagation test. Several studies illustrated in Table 4 conclude that $\lambda \mathrm{imm}^{\mathrm{P} 22}$ propagation in E. coli is mainly dependent on efficient ribosome rescue and that the inactivation of the tagging activity did not affect phage growth. It was also reported that the threshold SsrA function required for plaque formation in E. coli is fairly low [23]. Thus, the absence of phage $\lambda i m m^{\mathrm{P} 22}$ propagation in the E. coli $\Delta s s r A$ expressing wild type Hp-SsrA (that complements growth defect) 
was unexpected (Table 3). In contrast to Hp-SsrA, wildtype SsrA from Neisseria gonorrhoeae (NG-SsrA) restores phage propagation in E. coli $\Delta s s r A$ [20]. Interestingly, NG-SsrA mutant versions carrying mutations affecting either the ribosome rescue function (NG-SsrA UG) or the functionality of the tag sequence ( $S s r \mathrm{ADD}^{\mathrm{DD}}$ and SsrAOchre) were defective in complementing the phage propagation in $E$. coli $\triangle s s r A$. This suggests that under conditions of heterologous complementation of $E$. coli $\triangle s s r A$ either with Hp-SsrA (this work) or with NG-SsrA [20], $\lambda \mathrm{imm}^{\mathrm{P} 22}$ phage propagation requires trans-translation-dependent protein tagging in addition to ribosome rescue. The proposition of a secondary role of protein tagging in $\lambda \mathrm{imm}^{\mathrm{P} 22}$ propagation in $E$. coli is compatible with the observation by Withey and Friedman [14] that smaller plaques were generated in an E. coli strain expressing a $\mathrm{SsrA}^{0}$ mutant that encodes a truncated tag. They postulate that the tag is not necessary for phage propagation but is required to allow an optimal growth of phages.

\section{Conclusions}

To conclude, heterologous complementation showed that the wild type Hp-SsrA is able to restore normal growth to an $E$. coli $\triangle s s r A$ mutant suggesting that despite the sequence differences between these molecules, Hp-SsrA acts as a partially functional but not optimal tmRNA in $E$. coli. The tag sequence of Hp-SsrA presents several differences with that of the other studied bacteria, in particular a different resume codon, a charged residue at the end of the tag (Lysine instead of Leucine or Valine) (Figure 4) and the absence of a SspB protein recognition motif. We propose that these differences might account for the inability of the Hp-SsrA to support phage propagation in an $E$. coli $\triangle s s r A$ mutant. This attributes an additional role of trans-translational dependent tagging for efficient $\lambda$ $\mathrm{imm}^{\mathrm{P} 22}$ phage propagation in $E$. coli. Our interpretation is that this secondary role of protein tagging is revealed by heterologous complementation because ribosome rescue is less efficient. This emphasizes once again the regula-

Table 4: Phenotypes of the different mutants of $E$. coli ssrA

\begin{tabular}{|c|c|c|c|c|}
\hline $\begin{array}{l}\text { E. coli } \\
\text { SsrA version }\end{array}$ & Effects on SsrA & $\begin{array}{l}\text { SsrA tag appended to truncated } \\
\text { proteins }\end{array}$ & EOP§ & Reference \\
\hline SsrAWT & Wild type & ANDENYALAA & 1 & {$[14,15]$} \\
\hline SsrA & $\begin{array}{l}\text { Substitution of } \\
\text { the resume } \\
\text { codon by a stop } \\
\text { codon }\end{array}$ & None & $1.3 \times 10^{-5}$ & {$[14]$} \\
\hline SsrAwobble & $\begin{array}{l}\text { Absence of } \\
\text { alanylation of the } \\
\text { tRNA-like domain } \\
\text { of SsrA }\end{array}$ & None & $5 \times 10^{-5}$ & {$[28]$} \\
\hline SsrA $A^{S m p B}$ & $\begin{array}{l}\text { Absence of } \\
\text { interaction } \\
\text { between SsrA and } \\
\text { SmpB }\end{array}$ & None & N.D. & \\
\hline SsrADD & $\begin{array}{l}\text { Substitution of } \\
\text { the last two } \\
\text { alanine residues } \\
\text { of the tag } \\
\text { by two aspartate } \\
\text { residues }\end{array}$ & ANDENYALDD & $0.5--0.1$ & {$[28]$} \\
\hline SsrASTOP & $\begin{array}{l}\text { Two stop codons } \\
\text { added after } \\
\text { the resume codon }\end{array}$ & Minimal tag added & 0.9 & {$[14]$} \\
\hline
\end{tabular}

${ }^{\S}$ EOP is the ratio between the titer of phage on a lawn of bacteria expressing one of the indicated SsrA versions and the titer of phage on a wild type bacterial lawn; N.D.: Not determined. 
tory role of trans-translation in addition to its quality control function.

In conclusion, tmRNAs found in all eubacteria, have coevolved with the translational machinery of their host and possess specific determinants that were revealed by this heterologous complementation study.

\section{Methods}

\section{Bacterial strains and growth conditions}

Escherichia coli strain MG1655, MG1655 $\Delta s s r A[18]$ and MG1655 $\triangle s m p B$ [18] were grown at $37^{\circ} \mathrm{C}$ on solid or liquid LB medium. These strains were used as recipients for plasmids carrying different $H$. pylori genes:smpB, ssr $A$ and mutant versions of ssrA as well as the E. coli ssrA gene (Table 2). Both antibiotics chloramphenicol $(\mathrm{Cm})$ and spectinomycin $(\mathrm{Sp})$ were used at $100 \mathrm{\mu g} \mathrm{ml}^{-1}$ and isopropyl- $\beta$-D-thiogalactoside (IPTG) at $1 \mathrm{mM}$. H. pylori strain 26695 was grown under standard conditions, and harvested in mid-log phase as described in [10]. Doubling times ( $\mathrm{g}$ values) correspond to the mean generation time.

\section{Molecular techniques and sequencing}

Plasmids pILL788, pILL791, pILL792, pILL793, pILL794, pILL795, pILL2328 correspond to $H$. pylori $S s r A^{W T}$, $s s r A^{D D}, \quad s s r A^{\text {resume }}, \operatorname{ssr} A^{\text {wobble }}, \operatorname{ssr} A^{\text {smpB }}, \quad s s r A^{S T O P}$ genes cloned into the E. coli/H. pylori shuttle vector pILL2150 [24], respectively. SsrA mutagenesis has been described in [10]. The H. pylori ssrA gene amplified by PCR with primers H367 (5'-CGGGATCCCTCACCTGTTCTTTCTGA-3') and H368 (5'-GGGGTACCCGGATCCTT AATCGAATAAAAATCAGG-3') was cloned into the pEXT21 low copy number vector (1-3 copies per cell) [25] using BamHI/KpnI restriction sites (Table 1). The resulting plasmid was designated pILL2318.

The E. coli ssrA gene amplified by PCR with primers H365 5'-CTATCCCGGCGC TGGGTAACATCGGG-3, and H366 5'-GCTTTTCGTTGGGCCTATCAATGGGCC-3' was cloned into pILL2150, to generate pILL2334. The $H$. pylori smpB gene amplified by PCR with primers H225 (5'-GGACTAGTAGGAAGAGAAT AATGAAACTCATTGCCAG CAAC-3') and H236 (5'CGGGGTACCTTATCCTTTAAAGTGGTGTTT-

TAAATCAGC-3'), was cloned into pILL2150 [24] using SpeI/KpnI restriction sites to generate pILL786.

\section{Test of $\lambda$ imm $^{\mathrm{P} 22}$ propagation in E. coli}

The efficiency of plating (EOP) strains was determined by plating tenfold serial dilution of phage $\lambda \mathrm{imm}^{\mathrm{P} 22}$ on top agar mixed with $100 \mu \mathrm{l} E$. coli overnight liquid culture in $\mathrm{LB}$ with $0.4 \%$ maltose and $10 \mathrm{mM} \mathrm{MgSO}_{4}$. The number of CFU.ml-1 was calculated for each E. coli strain. The EOP is the ratio between the titer of phage on a bacterial lawn of the indicated strain (Table 3 ) and that of the wild type strain.

\section{Western blot}

Western blot to detect SmpB proteins was performed with $E$. coli whole cell sonicates prepared as in [26]. Protein concentrations were measured with Bradford assay (Bio-Rad). Twenty $\mu \mathrm{g}$ of crude extracts were separated by 15\% SDS-PAGE and blotted on a polyvinylidene difluororide membrane (PVDF, Millipore). Hp-SmpB and Ec$\mathrm{SpmB}$ were detected with rabbit polyclonal antibody raised against Ec-SmpB (a generous gift of B. Felden). Binding of the IgG anti-rabbit coupled peroxydase antibody (Amersham) was revealed with the ECL Plus reagent (Pierce).

\section{RNA extraction, riboprobe synthesis and northern blot}

RNAs were extracted using the phenol-chloroform method as described in [27]. An E. coli 5S rRNA riboprobe was synthesized using both primers H357 (5GCCTGGCGGCAGTAGCG CG GTGG-3') and H358 (5'-CTAATACGACTCACTATAGGGAGAGCCTGGCA GTTCCC TACTCTCGC-3'). Riboprobes synthesis for $H$. pylori SsrA was as in [10]. The ladder used corresponds to pBR322 vector digested by $M s p I$ and labeled at the 5 'end with $\gamma^{32}$ P ATP. Intensities of the bands were determined with Quantity One Software (Bio-Rad). The northern blot procedure was as described in [10].

\section{Authors' contributions}

Conceived and designed the experiments: MT, HDR. Performed the experiments: MT, SA, CE. Analyzed the data: MT, HDR. Wrote the paper: MT, HDR. All authors read and approved the final manuscript.

\section{Acknowledgements}

The authors thank A. Labigne for her support. We also want to thank B. Felden for the gift of anti-EcSmpB antibodies and for constructive comments. We are grateful to J. Collier and P. Bouloc for the gift of E. coli strains MG1655 $\triangle S S$ rA and $\triangle s m p B$ and to H. Neil, K. Zemam and C. Saveanu for experimental help. M. Thibonnier was supported by a FRM grant.

\section{Author Details}

1'Institut Pasteur, Unité P. Pathogenèse de Helicobacter, 28 rue du Dr. Roux, 75724 Paris Cedex 15 France, ${ }^{2}$ Current address: Institut Pasteur, Unité de Recherche et d'Expertise Bactéries anaérobies et Toxines, Paris, France and ${ }^{3}$ Current address: Institut Pasteur, G5 Biologie et Génétique de la Paroi Bactérienne, Paris, France

Received: 23 November 2009 Accepted: 26 March 2010

Published: 26 March 2010

References

1. Dulebohn D, Choy J, Sundermeier T, Okan N, Karzai AW: Trans-translation: the $t m$ RNA-mediated surveillance mechanism for ribosome rescue, directed protein degradation, and nonstop mRNA decay. Biochemistry 2007, 46:4681-4693.

2. Moore SD, Sauer RT: The tm RNA system for translational surveillance and ribosome rescue. Annu Rev Biochem 2007, 76:101-124.

3. Keiler KC: Biology of trans-Translation. Annu Rev Microbiol 2008, 62:133-151.

4. Keiler K, Waller P, Sauer RT: Role of a peptide tagging system in degradation of proteins synthesized from damaged messenger RNA. Science 1996, 271:990-993.

5. Gueneau de Novoa P, Williams K: The tm RNA website: reductive evolution of tmRNA in plastids and other endosymbionts. Nucleic Acids Research 2004, 1:D104-D108. 
6. Keiler KC: Physiology of tm RNA: what gets taggedand why? Curr Opin Microbiol 2007, 10:169-175.

7. Keiler KC, Shapiro L: tm RNA is required for correct timing of DNA replication in Caulobacter crescentus. J Bacteriol 2003, 185:573-580.

8. Lessner FH, Venters BJ, Keiler KC: Proteolytic adaptor for transfermessenger RNA-tagged proteins from a-proteobacteria. J Bacteriol 2007, 189:272-275.

9. Okan NA, Bliska JB, Karzai AW: A Role for the SmpB-SsrA system in Yersinia pseudotuberculosis pathogenesis. PLoS Pathogens 2006, 2:e6.

10. Thibonnier M, Thiberge J-M, De Reuse H: Trans-Translation in Helicobacter pylori: Essentiality of Ribosome Rescue and Requirement of Protein Tagging for Stress Resistance and Competence. PLOS ONE 2008, 3:e3810

11. Yang C, Glover JR: The SmpB-tm RNA tagging system plays important role in Streptomyces coelicolor growth anddevelopment. Plos One 2009, 4:e4459.

12. Komine Y, Kitabatake M, Yokogawa T, Nishikawa K, Inokuchi H: A tRNAlike structure is present in 10Sa RNA, a small stable RNA from Escherichia coli. Proc Nat Acad Sci 1994, 91:9223-9227.

13. Retallack DM, Johnson LL, Friedman DI: Role of 10Sa RNA in the growth of $\lambda$-P22 hybrid phage. Journal of Bacteriology 1994, 176:2082-2089.

14. Withey J, Friedman DI: Analysis of the role of trans-translation in the requirement of tmRNA $\lambda$ immP22 growth in Escherichia coli. J Bacteriol 1999:2148-2157.

15. O'Connor M: Minimal translation of the $t m$ RNA tag-coding region is required for ribosome release. Biochemical and Biophysical Research Communications 2007, 357:276.

16. Karzai WA, Susskind MM, Sauer RT: SmpB, a unique RNA-binding protein essential for the peptide-tagging activity of SsrA ( $t m$ RNA). EMBO J 1999, 18:3793-3799

17. Robinson $\mathrm{K}$, Argent $\mathrm{RH}$, Atherton JC: The inflammatory and immune response to Helicobacter pylori infection. Best Pract Res Clin Gastroenterol 2007, 21:237-259

18. Hallier M, Ivanova N, Rametti A, Pavlov M, Ehrenberg M, Felden B: Prebinding of small protein $B$ to a stalled ribosome triggers transtranslation. J Biol Chem 2004, 279:25978-25985.

19. Hutchison CA III, Peterson SN, Gill SR, Cline RT, White O, Fraser CM, Smith $\mathrm{HO}$, Craig Venter J: Global transposon mutagenesis and a minimal Mycoplasma genome. Science 1999, 286:2165-2169.

20. Huang C, Wolfgang MC, Withey J, Koomey M, Friedman DI: Charged tm RNA but not tmRNA-mediated proteolysis is essential for Neisseria gonorrhoeae viability. EMBO J 2000, 19:1098-1107.

21. Akerley BJ, Rubin EJ, Novick VL, Amaya K, Judson N, Mekalanos JJ: A genome-scale analysis for identification of genes required for growth or survival of Haemophilus influenzae. PNAS 2002, 99:966-971.

22. Williams KP, Marindale KA, Bartel DP: Resuming translation on tm RNA: a unique mode of determining a reading frame. the EMBO Journal 1999 18:5423-5433

23. Miller MR, Healey DW, Robison SG, Dewey JD, Buskirk AR: The role of upstream sequences in selecting the reading frame of $t m$ RNA. BMC Biol 2008, 6:29.

24. Boneca IG, Ecobichon C, Chaput C, Mathieu A, Guadagnini S, Prevost M-C, Colland F, Labigne A, de Reuse H: Development of inducible systems to engineer conditional mutants of essential genes of Helicobacter pylori. Appl Environ Microbiol 2008, 74:2095-2102.

25. Dykxhoorn DM, St Pierre R, Linn T: A set of compatible tac promoter expression vectors. Gene 1996, 177:133-136.

26. Cussac V, Ferrero R, Labigne A: Expression of Helicobacter pylori urease genes in Escherichia coli grown under nitrogen-limiting conditions. $J$ Bacteriol 1992, 174:2466-2473.

27. Bury-Moné S, Thiberge J-M, Contreras M, Maitournam A, Labigne A, De Reuse H: Responsiveness to acidity via metal ion regulators mediates virulence in the gastric pathogen Helicobacter pylori. Mol Microbiol 2004, 53:623-638.

28. Cheng Z, Deutscher M: Purification and characterization of the Escherichia coli exoribonuclease RNase R. Comparison with RNase II. Biol Chem 2002, 277:21624-21629.

doi: 10.1186/1471-2180-10-91

Cite this article as: Thibonnier et al., Study of the functionality of the Helicobacter pylori trans-translation components SmpB and SsrA in an heterologous system BMC Microbiology 2010, 10:91

\section{Submit your next manuscript to BioMed Central} and take full advantage of:

- Convenient online submission

- Thorough peer review

- No space constraints or color figure charges

- Immediate publication on acceptance

- Inclusion in PubMed, CAS, Scopus and Google Scholar

- Research which is freely available for redistribution 